\title{
Unsupervised Color Image Segmentation Based on Non Parametric Clustering
}

\author{
Imène Kirati and Yamina Tlili \\ Department of Computer Science, Univesity of Badji Mokhtar, Annaba, Algeria
}

\begin{abstract}
Many segmentation problems have been addressed using probabilistic modeling. These methods tend to estimate the region membership probabilities for each pixel of the image. The segmentation results depend strongly on the initialization of these regions and the selection of the appropriate number of segments. In this paper we present an unsupervised segmentation method based on non parametric clustering able to deal with these two issues. After a simple splitting, a minimum variance criterion is used to generate both the initial regions and their number. The proposed model was applied on various images (synthetic, natural) showing good visual results. Finally numerical experiments demonstrate the efficiency and the robustness of the proposed model compared to other segmentation methods.
\end{abstract}

Keywords: image segmentation, non parametric clustering, class initialization, homogeneity

\section{Introduction}

Image segmentation is one of the fundamental tasks in image processing and computer vision. This process aims to simplify and divide the image into meaningful regions making the object recognition and the interpretation simpler. The image pixels are grouped on regions with respect to homogenous local features (ex. color, intensity, texture...). Several survey papers related to image segmentation techniques have appeared in the literature [1], [2]. Nevertheless, there is no universal segmentation method that can be applied for all images and the elaboration of an image segmentation algorithm depends on the type of image and the field of application.

Many existing segmentation techniques are based on clustering, which is a powerful technique employed in diverse applications of image processing. Clustering algorithms [3] can be classified into four types: hard clustering, fuzzy clustering, hierarchical clustering and probabilistic clustering.

This paper deals with probabilistic clustering. This type of clustering models the image on a distribution where each component of this distribution represents a region of the image. These methods rely on the parametric and the non parametric modeling of distribution.

In the parametric models, regions are assumed to follow a basic distribution (ex. Gaussian, Poisson...), in this case, the goal of the clustering procedure is to determine the distribution parameters and assign each pixel to the component (region) that has the largest posterior probability.

Many Gaussian Mixture Model (GMM) [4] based approaches have been shown to be successful in image segmentation applications, see for instance [5], [6], [7], [8]. However, the distribution of the data is generally unknown and each cluster may follow its own distribution [9]. However, the parametric assumptions on the cluster distribution can be a limit and constraining all clusters to follow the same distribution can impose an inadequate shape for clusters.

The non parametric models overcome this restriction by using the data to estimate its own distribution and they do not impose a specific distribution to pixels. Determining the structure from using the data and not requiring an estimation of parameters are the strengths of these methods. 
To solve the image segmentation problem, Comaniciu et al. [10] suggested a non parametric model using generalized kernel approach. The iterative algorithm aims for finding modes i.e. local maxima of the estimated local density gradient of similar pixels. The set of image segments is constituted in function of these modes: pixels having the same modes are assigned to same segments. The algorithm described in [11] proposes a color image segmentation method by associating the non parametric estimation method with Gaussian kernel function, to a Bayesian classifier to classify image regions into foreground and background. In [12], a hybrid probabilistic framework for image segmentation is described where a non parametric kernel density estimate is combined with a GMM-EM model with the purpose to overcome the shortcoming of the parametric methods reported above.

However, in unsupervised probabilistic segmentation method, the results depend strongly on the initialization of regions. To achieve an optimal convergence, the choice of an initialization method is important and remains an open question.

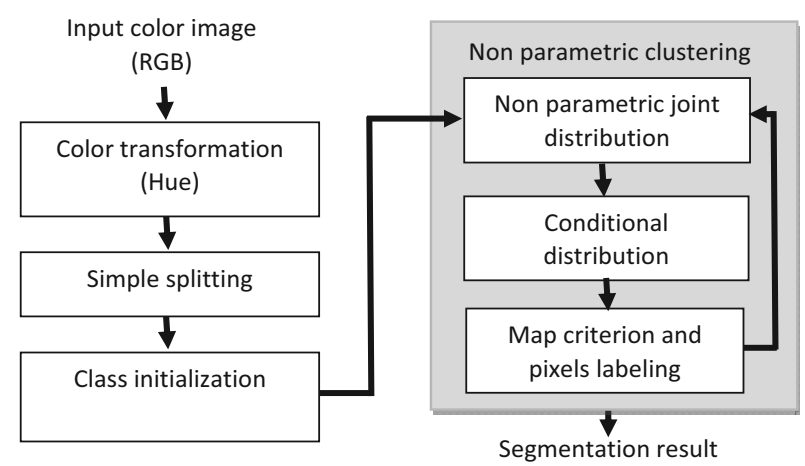

Figure 1. Outline of the proposed approach.

The initialization step can be done randomly; but in this case, the results are very sensitive and different at each initialization. Many models opt for using existing algorithms such as: K-means [13], Watershed [14] or Superpixels [15] [16] to perform the initialization of regions.

Another open question in image segmentation methods concerns the selection of the number of image regions. The large size of images and their complexity make the specification of an "appropriate" number of segments difficult. Many research papers have been published on unsupervised image segmentation [17], [18], [19]. These methods present algorithms that specify automatically the number of segments with different strategy.

The objective of this paper is to present a new unsupervised image segmentation method based on non parametric clustering. The proposed method has a number of advantages. First, the non parametric clustering can handle the complex data of the image (several variables per pixel). The model does not assume a prior distribution and the regions are generated by their own density function, this can be very interesting since the regions of the image are not usually of the same shape. Second, using a simple method based on homogeneity criterion, the proposed method is able to tackle the initialization problem on one hand and approximate the number of regions on the other.

The rest of the paper is organized as follows. In Section 2, the proposed model for image segmentation is described in detail. Qualitative and quantitative experiments on various images with comparison with existing segmentation methods are presented in Section 3. Finally, conclusions are given in Section 4.

\section{Segmentation Model}

The following section will explain in detail the steps of the proposed segmentation model illustrated in Figure 1.

\subsection{Color transformation}

The first step of our segmentation model is the color space transformation. The input image is transformed from RGB to HSV space. The HSV space is close to the human perception and, contrary to RGB space, the color information (hue) of pixel is represented independently from intensity and saturation. This would also be worthwhile because we will use only the quantified hue information to reduce the computational complexity and processing time. Using the hue information instead the RGB components has many advantages [20]:

- It can represent subjective color suitable for human observers, 
- The final result has less regions than by using RGB,

- The segmentation is performed on one dimension.

\subsection{Class initialization}

The second step aims to generate the initial regions and to specify their number. Here, we propose a simple method inspired by K-means algorithms where each class is represented by its centroid. To generate the initial centroids, we use the variance as a measure of homogeneity. The details of the steps are as follows:

1. Initially, the image is divided into rectangular blocks of size $5 \times 5$,

2. Compute the homogeneity $V$ of each block,

3. Keep only blocks with $V=0$ and compute the corresponding mean of hue value $M$,

4. Sort the means in ascending order and delete duplicate value, if any,

5. Set the counter s to one $(s=1)$ and the first centroid $C_{0}=M_{0}$,

6. If dist $\left(M_{s}, M_{s-1}\right)>T$ then $C_{s}=M_{s}$,

7. $s=s+1$ and repeat 6 until all $M_{s}$ are treated.

The number of centroids $C_{s}$ will represent the number of regions $\mathrm{K}$.

In addition to setting the threshold of variance to zero, we use blocks to spot neighborhood pixels with high homogeneity where each centroid is represented by the median of local adjacent pixel having the same hue. In this way, the algorithm tends to generate regions with high homogeneity.

\subsection{Non parametric clustering}

The last and the main step of our approach is to perform the non parametric clustering. This step aims to compute the class membership probabilities of pixels and assign to each one a class label $\mathrm{K}$.

Let $x_{n}=(h, i, j)$ be the vector representing the $n^{\text {th }}$ pixel; $n=1, \ldots, N$; where $N$ is the total number of pixels of the image and $h$ represents the hue value of the corresponding pixel. Following the idea of [17] [21], the spatial location $i$ and $j$ of pixels are added to the vector of information in order to apply spatial constraints. In fact, the clustering-based segmentation methods have the particular characteristic that the spatial information should be considered where it is intuitively most likely that the spatially adjacent pixels belong to the same cluster.

Many research papers agree that this prior information brings improvement to the segmentation results.

The clustering process starts with computing the non parametric joint distribution $f\left(x_{n}, k\right)$ needed for calculating the non parametric conditional distribution using in [22]:

$$
f\left(x_{n}, k\right)=\frac{\sum_{m=1}^{N} g_{\gamma}\left(x_{n}, x_{m}\right) A(m)}{\sum_{y=1}^{N} \sum_{z=1}^{N} g_{\gamma}\left(x_{y}, x_{z}\right)}
$$

where $A(m)$ is an indicator function that takes 1 as a value if $x_{m} \in k$ and 0 otherwise and $g_{\gamma}$ is a Gaussian kernel of width $\gamma$ :

$$
g_{\gamma}\left(x_{n}, x_{m}\right)=\frac{1}{2 \pi \gamma^{2}} e^{-\frac{2\left(x_{n}-x_{m}\right)^{2}}{\gamma^{2}}}
$$

Since $x_{n}, x_{m}$ are represented by vectors, we use the Euclidian distance $\Delta\left(x_{n}, x_{m}\right)$ to compute the difference between the $n^{\text {th }}$ and the $m^{\text {th }}$ pixels.

The formula (1) presented above requires a class initialization. To avoid this drawback, we propose to represent classes by their centroids. The joint distributions are now computed by:

$$
f\left(x_{n}, k\right)=\frac{g_{\gamma}\left(x_{n}, C_{k}\right)}{\sum_{y=1}^{N} \sum_{z=1}^{K} g_{\gamma}\left(x_{y}, x_{z}\right)}
$$

where $C_{k}$ represents the centroid of the class $k$.

Contrary to the previous formula (1) where all pixels of the class $k$ are used to compute the joint distribution, the formula (3) uses only the centroid of the class. In addition, to improve the initialization step, the use of centroids will be beneficial in terms of reducing complexity and time processing.

The prior probabilities $\pi_{k}$ are obtained by marginalizing the joint distribution. Then the conditional probabilities $P\left(k \mid x_{n}\right)$ can be computed 
from the joint and prior probabilities, using the Bayesian theorem:

$$
P\left(k \mid x_{n}\right)=\frac{\pi_{k} f\left(x_{n}, k\right)}{\sum_{y=1}^{K} \pi_{y} f\left(x_{n}, y\right)}
$$

Finally, we adopt the Maximum a Posteriori criterion (MAP) to assign to each pixel a class label $k$ :

$$
P\left(k \mid x_{n}\right)=\max _{k}\left\{P\left(k \mid x_{n}\right)\right\}
$$

In other words, pixels are assigned to the cluster $k$ that maximizes its conditional probability. This step achieves the clustering and all pixels are grouped in classes. The centroids of the new clusters are recalculated and the whole clustering process is reiterated until the values of the centroids get stabilized.

For the sake of clearness, the whole non parametric clustering algorithm can be summarized by the following pseudo-code sequence:

\footnotetext{
Input: Image ( $\mathrm{N}$ pixels), generated centroids;

Output: labeled pixels;

Begin

1. While centroids changed do

2. Compute the joint distributions $f\left(x_{n}, k\right)$ and the probabilities $\pi_{k}$,

3. Compute the conditional distributions $P\left(k \mid x_{n}\right)$,

4. Apply Map criterion and label pixels,

5. Recalculate the centroids of the new classes, 6. End_While
}

End.

\section{Experimental Results}

In this section, we conduct experiments on a variety of images to examine the effectiveness of the proposed image segmentation algorithm.

\subsection{Synthetic color images}

In the first experiment, we demonstrate the effectiveness of the proposed model to approximate the number of segments. For this purpose, three synthetic color images of different complexity were created of size $200 \times 200$ with a number of regions equal to 2, 4, 6 for the images $\mathrm{a}, \mathrm{b}, \mathrm{c}$ respectively. To make the segmentation more difficult, the images are degraded with Gaussian noise.

The results provided by the proposed algorithm are illustrated in Figure 2. As it can be observed, the method is robust against noise and generates a good segmentation. An evaluation with the correct classification rate (CCR) is also performed and the obtained scores are noted in Table 1.

The first image \#a is a simple two class image. The number of regions is well approximate and the rate of correct classification is $100 \%$ in the absence of noise and $98 \%$ in the presence of noise.
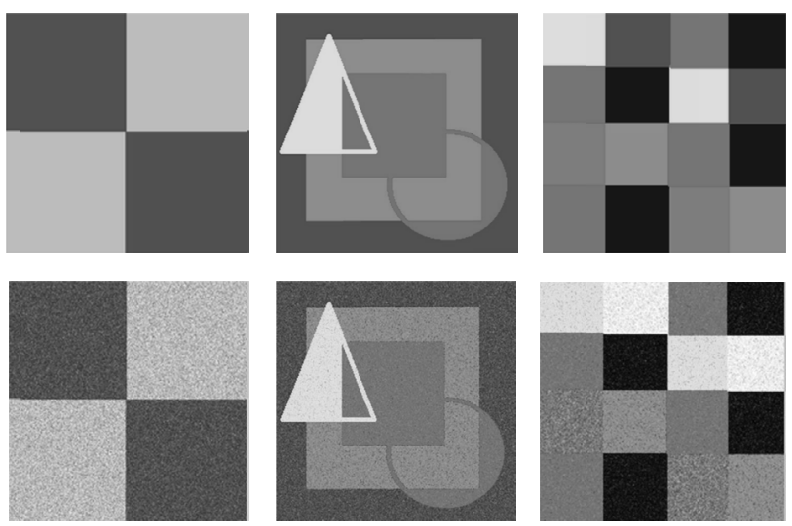

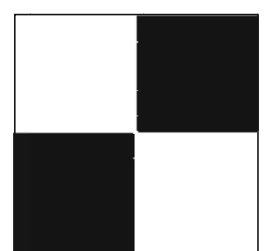

(a)

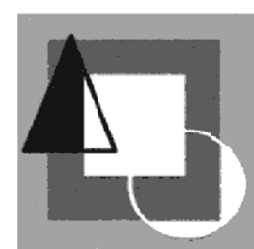

(b)

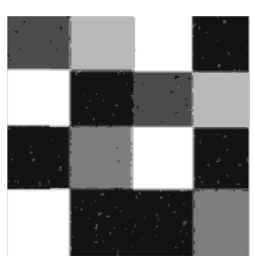

(c)
Figure 2. Segmentation results of synthetic images corrupted by Gaussian noise.

The second image \#b contains four overlapping regions. The number of segments is also well approximate and the rate of correct classification is $100 \%$ in the absence of noise and $94 \%$ in the presence of noise. 


\begin{tabular}{|c||c|c|c|c|}
\multicolumn{1}{|c||}{} & \multicolumn{2}{c|}{$\begin{array}{c}\text { Without Gaussian } \\
\text { noise }\end{array}$} & \multicolumn{2}{c|}{$\begin{array}{c}\text { With Gaussian } \\
\text { noise }\end{array}$} \\
\cline { 2 - 5 } & $\begin{array}{c}\text { Estimated } \\
\mathbf{K}\end{array}$ & $\begin{array}{c}\text { CCR } \\
(\boldsymbol{\%})\end{array}$ & $\begin{array}{c}\text { Estimated } \\
\mathbf{K}\end{array}$ & $\begin{array}{c}\text { CCR } \\
(\boldsymbol{\%})\end{array}$ \\
\hline \hline Image \#a & 2 & 100 & 2 & 98 \\
\hline Image \#b & 4 & 100 & 4 & 94 \\
\hline Image \#c & 5 & 86 & 5 & 81 \\
\hline
\end{tabular}

Table 1. Average performance of our algorithm on the BSDS, in terms of PR measure.

As illustrated, the third image \#c has 6 regions. The rate of correct classification is $86 \%$ in the absence of noise and $81 \%$ in the presence of noise. The number of segments generated is 5 , while the correct number of regions is 6 . We can perceive that the black and the gray pixels regions are assigned to the same class for the simple reason that, in the absence of saturation, the two colors have the same hue value.

\subsection{Natural color images}

The second experiment is conducted on real color images. These images were kept from the Berkeley Segmentation Database (BSDS) ${ }^{1}$ [23] which consists of 300 natural color images of diverse scene categories (200 training images and 100 test images). For each image, five to eight ground truths obtained from human-hand segmentation are available.

For all cases, the images are normalized to be of size $480 \times 320$ or $320 \times 480$ and each pixel is represented by a vector containing its hue measurement and its spatial location. The parameter $\gamma$ is determined empirically and was fixed to 0.1 .

To quantify the segmentation results, we use the probabilistic rand index (PRI). This measure counts the fraction of pairs of pixels whose labels are consistent with the computed segmentation and the ground truth averaging across multiple ground truth segmentations to account for scale variation in human perception [24]. The PR index between two segmentations map is then quantified as:

$$
P R(S, G)=\frac{2}{N(N-1)} \sum_{\substack{i, j \\ i<j}}\left[c_{i j} p_{i j}+\left(1-c_{i j}\right)\left(1-p_{i j}\right)\right]
$$

where $c_{i j}=1$ if the pixel $i$ and $j$ belong to the same region, $p_{i j}$ is the ground truth probability that pixels $i$ and $j$ belong to the same cluster, $N$ is the number of image pixels, $S$ is the computed segmentation results and $G$ is a set of given manually labeled images. The value of the PR index ranges between 0 and 1 . For example, if a PR measure is equal to 0.54 , this means that, compared to the ground truth image, on average $54 \%$ of pairs of pixel labels are correctly classified. In more exact terms, the closer the PR index value is to one, the better the segmentation result is.

Table 2 displays the obtained segmentation results on six randomly chosen images from the BSDS. The first column presents the original color images and the second presents the visual segmentation results where each region is represented by the mean of its color. The third and fourth columns present the estimated number of segments $\mathrm{K}$ and the PRI computed for each result.

As shown in the second column of Table 2, the proposed method yields a good perceptual segmentation results and we can observe the homogeneity of the obtained regions and also the accuracy and regularity of their boundaries.

The quantitative evaluation based on the PRI confirms that the segmentation results are close to manual segmentation where we can see high scores of correct classification. Despite this, we notice that the image \#181018 subscribe the lowest score, which is due to closeness of the hue value of pixels in the hat, jacket and wall regions. Thus, as demonstrated in the experiment on synthetic images above, the absence of saturation may decrease the quality of segmentation.

We consider also the computational cost of the proposed method. A PC, which is equipped with a 2.0-GHz Intel core duo $\mathrm{CPU}$ and 2-GB memory, is used.

The last column in Table 2 highlights the computational cost of each result. We can see that in all cases the algorithm runs in less than 2 seconds. The complexity time depends also on the size and the generated number of classes.

\footnotetext{
${ }^{1}$ http://www.eecs.berkeley.edu/Research/Projects/CS/vision/grouping/segbench/
} 


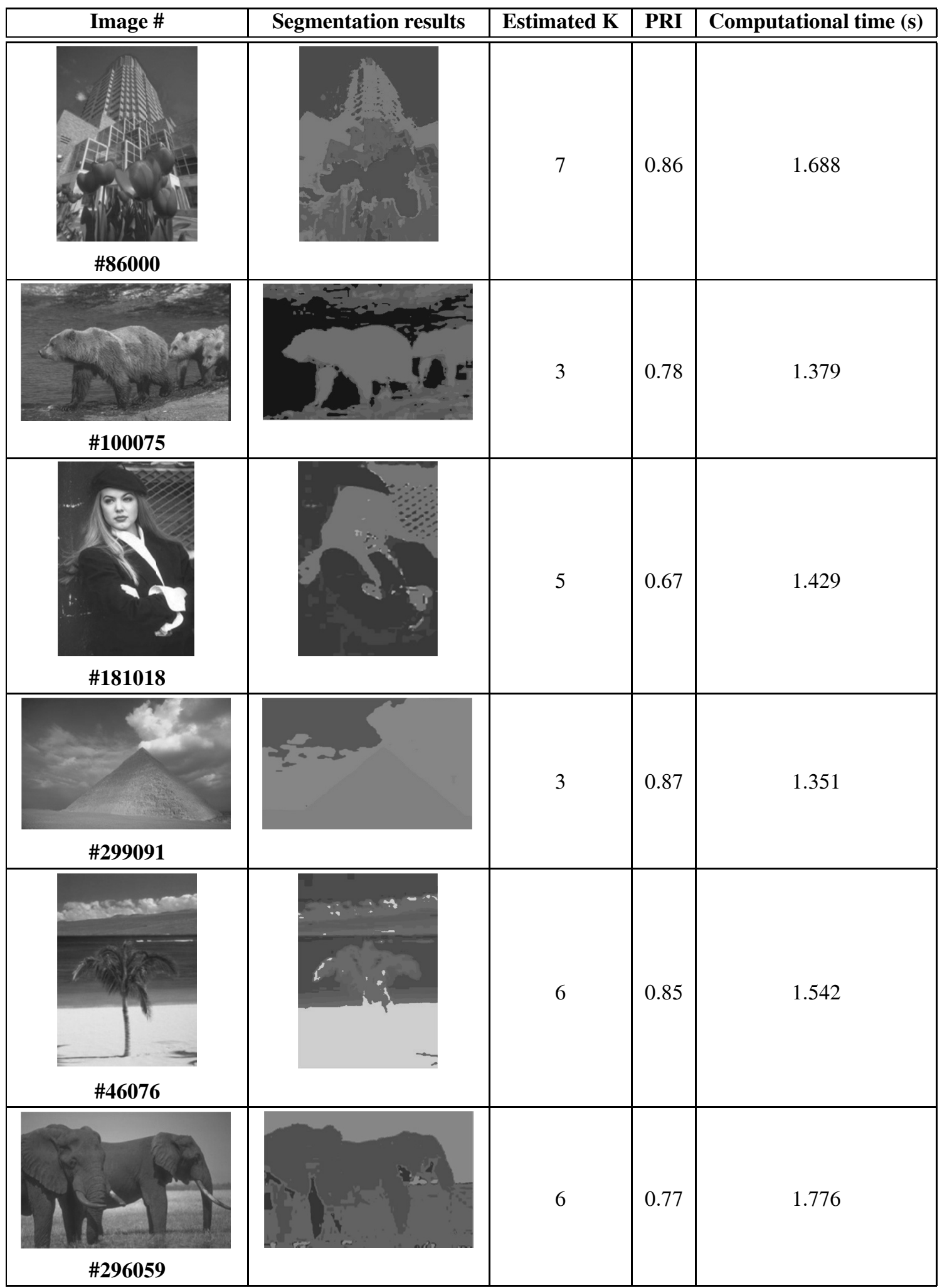

Table 2. Image segmentation results from the Berkeley segmentation database.

Finally, our method is compared to some of the recent color segmentation methods proposed in the literature: FCR [25], Mean Shift [10], N-
Cuts [26], DCM [27] and JSEG [28]. Figure 3 depicts the mean value of performance measure over the BSDS of the methods cited above. 
From Figure 3, we can see that our method achieved a score of $76 \%$ of correct classification, which is very close to the score achieved by the JESG algorithm; the two methods take the third place after FCR and Mean Shift. Furthermore, it outperforms widely the N-Cuts and DCM methods.

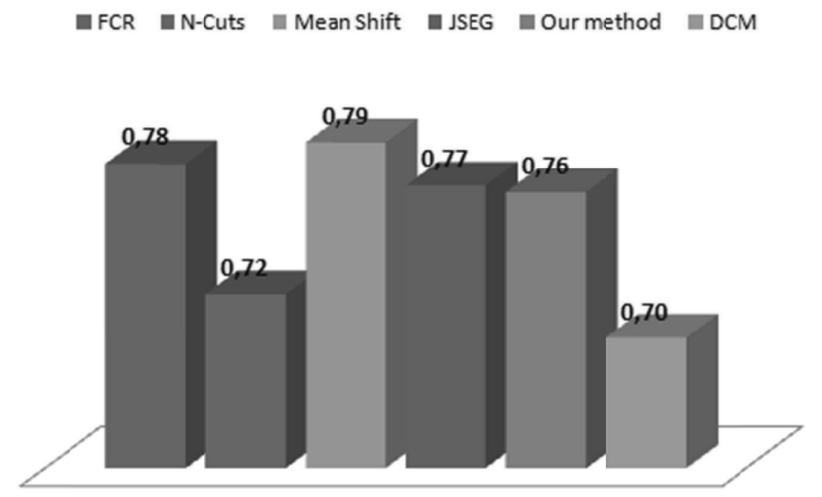

Figure 3. Quantitative comparison of our results with other methods used on the BSDS with PRI.

\section{Conclusion}

In this paper we have presented a new segmentation model based on probabilistic clustering. This unsupervised model exploits the robustness of the non parametric clustering. Further, it has the capacity to generate an initialization of classes and approximate the number of segments, using a simple strategy based on the homogeneity of regions. Experiments made on a variety of images highlight the validity of the proposed segmentation schema. Results on synthetic images confirm its potential of specifying an appropriate number of regions. The method is also successfully applied on Berkeley image database, showing good visual results. The comparative experimentation is promising where it performs competitively among the recently reported state-of-the-art segmentation methods. Moreover, it requires a low computational complexity suitable for real-time image segmentation processing.

However, experiments show that, in absence of saturation, our method may assign pixels of different regions to the same class when their hues are too close. We think also that adding other pixel descriptors like texture could bring refinement to the results. Future work will investigate these issues.

\section{References}

[1] N. R. PAL, S. K. PAL, A review on image segmentation techniques, pattern recognition. Elsevier, 26 (1993), 1277-1294.

[2] R. DASS, PRIYANKA, S. DEVI, Image Segmentation Techniques. International Journal of Electronics \& Communication Technology, 3(1) (2012), 66-70.

[3] R. XU, D. Wunsch II, Survey of Clustering Algorithms. IEEE Transactions on Neural Networks, 16 (2005), 645-676.

[4] M. A. T. Figueiredo, A. K. JAIN, Unsupervised learning of finite mixture models. Transactions on Pattern Analysis and Machine Intelligence, 24 (2002), 381-396.

[5] Z. Ji, Y. XiA, Q. Sun, Q. Chen, D. XiA, D. D. FenG, Fuzzy local Gaussian mixture model for brain MR image segmentation. IEEE Transactions on Information Technology in Biomedicine, May; 16(3) (2012-08-2), 339-347.

[6] M. Sujaritha, S. Annadurai, Color Image Segmentation using Adaptive Spatial Gaussian Mixture Model. International Journal of Information and Communication Engineering, 6 (2010), 28-32.

[7] F. CHEN, K. TANKA, T. HORIGUCHI, Image Segmentation Based on Bethe Approximation for Gaussian Mixture Model. Interdisciplinary Information Sciences, 11(1) (2005), 17-29.

[8] A. Peñalver, F. Escolanoet, J. M. SÁEz, Color Image Segmentation Through Unsupervised Gaussian Mixture Models. Advances in Artificial Intelligence, SpringerLink, Volume 4140/2006, (2006), 149-158.

[9] P. K. Mallapragada, R. Jin, A. Jain, Nonparametric Mixture Models for Clustering. International conference on structural, syntactic, and statistical pattern recognition, (2010), SpringerVerlag Berlin, Heidelberg.

[10] D. Comaniciu, P. Meer, Mean Shift: A Robust Approach toward Feature Space Analysis. IEEE Transaction on Pattern Analysis and Machine Intelligence, 24 (2002), 603-619.

[11] H. RAHIMIZADEH, M. H. MARHABAN, R. M. KAMIL, N. B. ISMAIL, Color Image Segmentation Based on Bayesian Theorem and Kernel Density Estimation. European Journal of Scientific Research, 26(3) (2002), 430-436.

[12] M. Andreetto, L. Zelnik-Manor, P. Perona, Non-Parametric Probabilistic Image Segmentation. Presented in the Proceedings of the IEEE 11th International Conference on Computer Vision, ICCV, (October 2007), 14-20, Rio de Janeiro, Brazil. 
[13] M. ZRibi, M. Benjelloun, A. Ben Hamida, A kMeans Clustering Algorithm Initialization for Unsupervised Statistical Satellite Image Segmentation. Presented in the Procedings of the First IEEE International Conference on E-Learning in Industrial Electronics, (2006), 11-16, France.

[14] S. Makrogiannis, G. Economou, S. FotopouLOS, A region dissimilarity relation that combines feature-space and spatial information for color image segmentation. IEEE Systems, Man, and Cybernetics, Part B: Cybernetics, 35(1) (2005), 44-53.

[15] A. Y. YANG, J. Wright, S. SASTRY, Y. Ma, Unsupervised Segmentation of Natural Images via Lossy Data Compression. Computer Vision and Image Understanding, 110(2) (2008), 212-225.

[16] J. Cheng, J. LiU, Y. Xu, F. Yin, D. W. Wong, B. H. LeE, C. Cheung, T. Aung, T. Y. Wong, Superpixel classification for initialization in model based optic disc segmentation. Presented in the Procedings of the International Conference of the IEEE Engineering in Medicine and Biology Society, (2012), 1450-1453.

[17] C. Constantinopoulus, A. LiKas, Image modeling and segmentation using incremental Bayesian mixture models. Computer Analysis of Images and Patterns, 4673 (2007), 596-603.

[18] P. Guo, C. C. Cheung, L. XU, Region number determination in automatic image segmentation based on BKYY model selection criterion. Presented in the Procedings of the IEEE-EURASIP Workshop on Nonlinear Signal and Image Processing, (June 1999), 743-746, Antalya, Turkey.

[19] J. Fan, D. Yau, A. Elmagarmid, W. Aref, Automatic image segmentation by integrating coloredge extraction and seeded region growing, IEEE Transaction on Image Processing, 10(10) (2001), 1454-1466.

[20] H.-D. Cheng, Y. Sun, A Hierarchical Approach to Color Image Segmentation Using Homogeneity. IEEE Transaction on image processing, 9(12) (2000), 2071-2082.

[21] C. Carson, S. Belongie, H. Greenspan, J. Malik, Blobworld: Image segmentation using expectationmaximization and its application to image querying. IEEE Transactions on Pattern Analysis and Machine Intelligence, 24 (2002), 1026-1038.

[22] G. Bougenière, C. Cariou, K. Chehdi, A. Gay, Non Parametric Stochastic Expectation Maximization for Data Clustering. Communications in Computer and Information Science, Springerlink, 23 (2009), 293-303.

[23] D. Martin, C. Fowlkes, D. Tal, J. Malik, A database of human segmented natural images and its application to evaluating segmentation algorithms and measuring ecological statistics. Presented in the Proceedings of the 8th Int. Conf. Computer Vision, 2 (July 2001), 416-423.
[24] R. Unnikrishnan, C. Pantofaru, M. Hebert, A Measure for Objective Evaluation of Image Segmentation Algorithms. Presented in the Proceedings of CVPR Workshop Empirical Evaluation Methods in Computer Vision, (1967).

[25] M. MignotTe, Segmentation by fusion of histogram-based k-means clusters in different color spaces. IEEE Transaction on Image Processing, 17(5) (2008), 780-787.

[26] J. SHI, J. MALIK, Normalized cuts and image segmentation. IEEE Transactions on Pattern Analysis and Machine Intelligence, 22(8) (2002), 888-905.

[27] C. Nikou, A. C. Likas, N. P. Galatsanos, A Bayesian Framework for Image Segmentation with Spatially Varying Mixtures. IEEE Transaction on Image Processing, 19 (2010), 2278-2289.

[28] Y. Deng, B. S. Manjunath, Unsupervised segmentation of color texture regions in images and video. IEEE Transactions on Pattern Analysis and Machine Intelligence, 23(8) (2001), 800-810.

Received: May, 2013

Revised: October, 2013 Accepted: November, 2013

Contact addresses: Imène Kirati Department of computer science Univesity of Badji Mokhtar Annaba Algeria

e-mail: imen.kirati@univ-annaba.org

Yamina Tlili Department of computer science Univesity of Badji Mokhtar Annaba Algeria

e-mail: yamina.tlili@univ-annaba.org

IMÈNE KIRATI is a PhD student at Badji Mokhtar University Annaba Algeria and she has been preparing her $\mathrm{PhD}$ thesis at the Laboratory of Research in Computer Science (LRI) since 2010. Her PhD research is Bayesian approaches to unsupervised color image segmentation. Her main research interests are in computer vision, pattern recognition and image segmentation.

YAMINA TLILI holds a PhD degree in computer sciences mention artificial intelligence and language processing and she is a lecturer in the Department of Computer Sciences at Badji Mokhtar University Annaba Algeria since 1985. She is head of the project entitled "Documents indexing and security". She is an active researcher in texts, images and opinions mining, with the focus on applications in language processing and artificial intelligence. She has published a number of articles in international journals and conferences on these subjects. 